\title{
The study of the electromagnetic anomalies linked with the Earth's crustal activity in the frequency band $[0.001 \mathrm{~Hz}-100 \mathrm{kHz}]$
}

\author{
P. Palangio, C. Di Lorenzo, F. Masci, and M. Di Persio \\ Istituto Nazionale di Geofisica e Vulcanologia, Italy \\ Received: 28 June 2007 - Revised: 3 September 2007 - Accepted: 3 September 2007 - Published: 6 September 2007
}

\begin{abstract}
The technological and the scientific objectives of the MEM (Magnetic and Electric fields Monitoring) project concerning to the study of the electromagnetic signals linked with the Earth's crustal activity are reported. The MEM project has been activated in Central Italy to create a network of observatories so as to monitoring the electromagnetic signals, both natural and artificial, in the frequency band $[0.001 \mathrm{~Hz}-100 \mathrm{kHz}]$. Some examples of the developed instrumentation and the know-how transfer to the industry are reported. We also report some results obtained in the first MEM station installed in the area of the INGV (Italian Istituto Nazionale di Geofisica e Vulcanologia) Observatory of L'Aquila. Using the single station magnetotelluric approach we have obtained some valuables information about the underground resistivity structure in the area of the measurement station. Concerning to the study of the magnetic signals linked with the tectonic activity we have reported an example of the long term behaviour of the magnetic induction vectors characteristics in the lower frequency band $[0.001-0.5] \mathrm{Hz}$, showing their normal behaviour when no large crustal changes due to geodynamical processes are present.
\end{abstract}

\section{Introduction}

The electromagnetic monitoring of the geodynamical processes is based on two different aspects. The first point is related to the study of the Earth's crust electric conductivity changes due to the tectonic stress variations of the rocks in the seismo-genetic area (Teisseyre and Ernst, 2002). The second point concerns the monitoring of the electromagnetic fields generated by the different mechanisms of the electromechanic energy conversion. These mechanisms depend on the rocks characteristics (Molchanov et al., 1995; Frid et al., 2000). The first point can be investigated by the long term monitoring of the magnetic transfer functions and the elements of the impedance tensor (Spitz, 1985). The starting point of this research is based on the knowledge of the electric properties of the materials present in the focal zone of the earthquake by means of the Earth's conductivity structure estimation. Concerning to the second point we have to consider that the observed signal is due to the convolution of the source time functions and the Earth's impulse response functions. The knowledge of these response functions is a fundamental point for this kind of investigations. Another aspect concerns the structure of the natural and the artificial electromagnetic fields in the Earth-ionosphere cavity that change in a complex way in the space and the time domains. The measured signals on the Earth's surface are given by the overlap of a myriad of waves coming to the measurement point. Some studies report magnetic anomalies related to the seismic activity. The frequency band of these observations is extremely wide; it is extended from $\mathrm{mHz}$ to $\mathrm{MHz}$ (Molchanov et al., 1992). The [0.001-1000] Hz band can be related with linear processes (Molchanov and Hayakawa, 1995), while the [10-50] kHz band can be related to non linear processes in the electro-mechanic energy conversion (Parrot et al., 1993). In any case, taking into account the attenuation of the crossed medium, it is reasonable to observe electromagnetic signals in the band [0.001-1000] Hz. So, this frequency band can be considered the interval in which to focalize the research activity as, according to some conditions (Kawate et al., 1998), the amplitude of the electromagnetic signals reaching the Earth's surface could be sufficient to discriminate it from the background noise (Johnston, 1984).

Correspondence to: F. Masci

(masci@ingv.it)

Published by Copernicus Publications on behalf of the European Geosciences Union. 


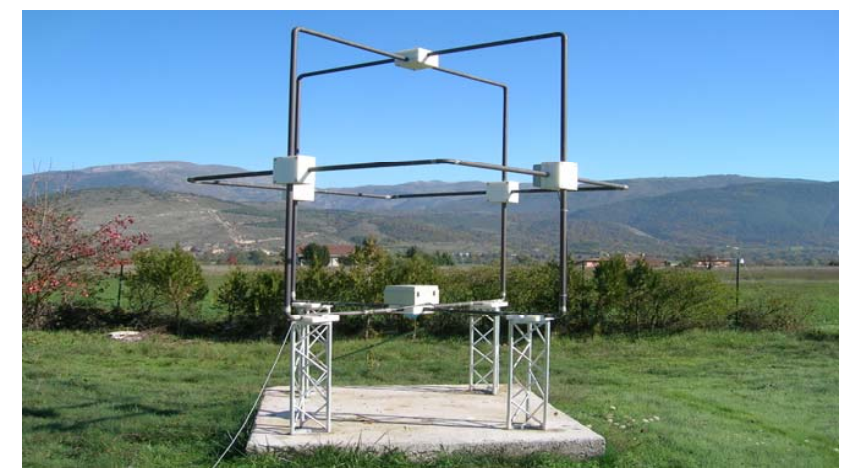

Fig. 1. Three axes induction antenna for magnetic field measurement in the frequency band $[1 \mathrm{~Hz}-100 \mathrm{kHz}]$. The instrumental sensitivity is equal to $1 \mathrm{fT} / \sqrt{\mathrm{Hz}}$.

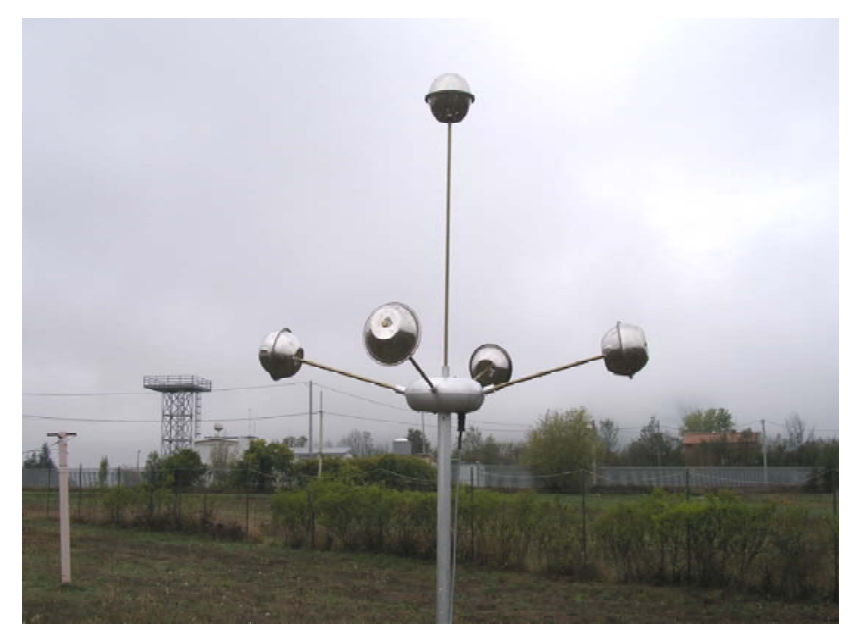

Fig. 2. Three axes antenna for electric field measurement in the frequency band $[1 \mathrm{~Hz}-100 \mathrm{kHz}]$. The instrumental sensitivity is equal to $1 \mu \mathrm{V} /(\mathrm{m} \sqrt{\mathrm{Hz}})$.

\section{The MEM Project}

The MEM Project (Interreg IIIA Adriatic Cross Border Programme) has been activated in the INGV Observatory of L'Aquila since 2004 (Palangio et al., 2007a ${ }^{1}$ ). The leader partner of the project is the Italian Abruzzo Region. The other partners are the INGV Observatory of L'Aquila, the Regional Environmental Agency of Molise Region (ARPAMolise), Italy, the University of Ferrara, Italy, the University of Tirana, Albania and the Geomagnetic Institute of Grocka, Beograd, Serbia. The main target of the MEM project is to realize a network of permanent observatories in Central Italy to monitoring the environmental electromag-

\footnotetext{
${ }^{1}$ Palangio, P., Di Lorenzo, C., Di Persio, M., Masci, F., Mihajlovic, S., Santarelli, L., and Meloni, A.: Electromagnetic monitoring of the Earth's interior in the frame of MEM project, submitted, Ann. Geoph-Italy, 2007a.
}

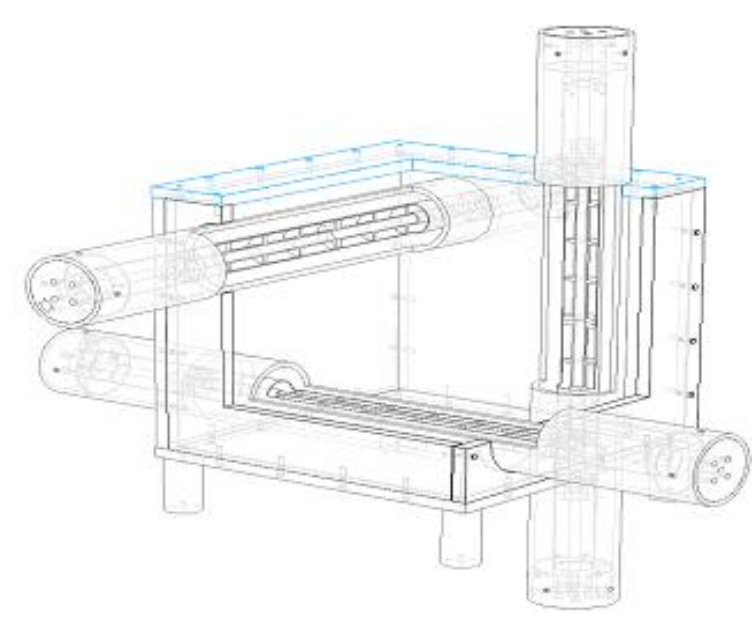

Fig. 3. Three-axes search coil magnetometer for magnetic field measurements in the frequency band $[0.001-100] \mathrm{Hz}$. The instrumental sensitivity is equal to $50 \mathrm{fT} / \sqrt{\mathrm{Hz}}$.

netic signals. Data collected in the network stations will allow us to realize environmental electromagnetic tomography by the representation of the electromagnetic fields distribution in the time, frequency and space domains as final target. Each station consists of two independent systems working in the two frequency bands $[0.001-25] \mathrm{Hz}$ and $[1 \mathrm{~Hz}-$ $100 \mathrm{kHz}$. In the first band the sampling rate is $100 \mathrm{~Hz}$ with anti-aliasing 8 poles low pass filter at $25 \mathrm{~Hz}$, whereas in the second band the sampling rate is $200 \mathrm{kHz}$ with notch filters at $50 \mathrm{~Hz}, 100 \mathrm{~Hz}$ and $150 \mathrm{~Hz}$. One of the scientific purposes of the MEM project is the electromagnetic monitoring of the geodynamical processes related to the tectonic activity. The other objectives are the characterization of the Earthionosphere cavity electromagnetic background noise in the $[0.001 \mathrm{~Hz}-100 \mathrm{kHz}]$ frequency band, the model realization of the Earth's crust electrical conductivity structure, and the study of the electromagnetic signals produced in the Earth's interior. The technological objectives of the project are the development of new instruments and the know-how transfer to the industry. In the first two years of the project new instruments have been developed and their industrialization has been realized by the Italian Geomagnetic System industry. In the Earth-ionosphere cavity there are a myriad of electromagnetic natural and artificial signals produced by a huge number of sources (Palangio, 1993). The spectrum of these signals spreads twelve orders of magnitude in the frequencies domain, and fourteen orders of magnitude in the energy domain (Lanzerotti et al., 1990). Taking into account the multiplicity of the parameters that must be measured, and the complexity of the involved physical mechanisms, the level of the instrumental noise needs to be so low to detect the weakest electromagnetic natural signal. The spectral density of the feeblest magnetic natural signal is about [5-10] $f T / \sqrt{\mathrm{Hz}}$ above the 


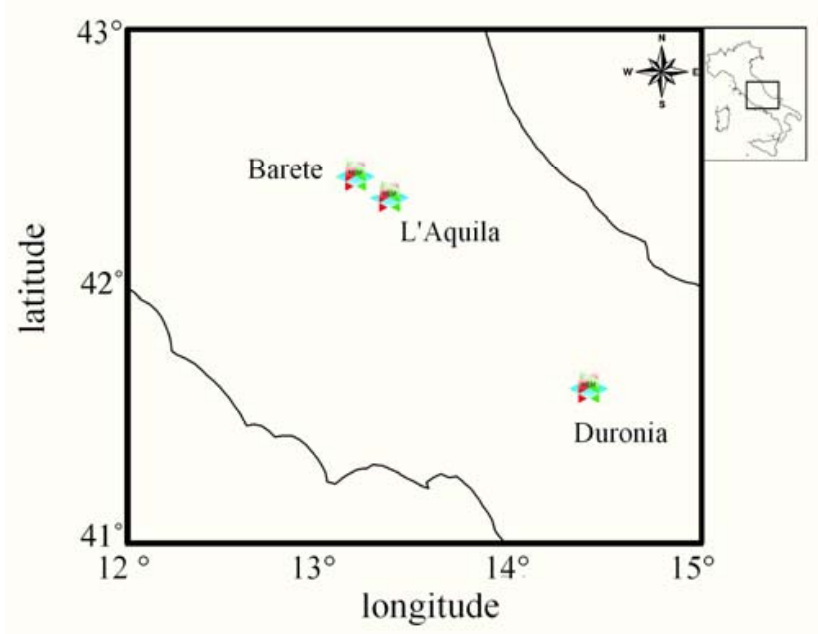

Fig. 4. The distribution of the first three MEM stations in Central Italy.

Earth-ionosphere cut-off frequency $(1.7 \mathrm{kHz})$, so the background noise level of the instrumentation must be at least [12] $f T / \sqrt{H z}$. In Figs. 1, 2 and 3 are shown some examples of the electric and magnetic sensors developed in the frame of the MEM project. These sensors have been installed in the first MEM station located in the area of L'Aquila INGV Geomagnetic Observatory $\left(42^{\circ} 23^{\prime} \mathrm{N}, 13^{\circ} 19^{\prime} \mathrm{E}, 682 \mathrm{~m}\right.$ a.s.1.). Within the end of 2007 two new MEM observatories will be installed in Barete $\left(42^{\circ} 30^{\prime} \mathrm{N}, 13^{\circ} 16^{\prime} \mathrm{E}, 930 \mathrm{~m}\right.$ a.s.1.) and Duronia $\left(41^{\circ} 39^{\prime} \mathrm{N}, 14^{\circ} 28^{\prime} \mathrm{E}, 910 \mathrm{~m}\right.$ a.s.l.). In Fig. 4 are reported the locations of the first three MEM stations in Central Italy. In each network station will be measured the three components of the magnetic field, the three components of the electric field and the two components of the telluric field. The network configuration is needed to separate the natural electromagnetic fields from the artificial ones and to separate the electromagnetic fields originated in the Earth's interior from those generated by an external source. When all the stations will be in operation the wide-band interferometry technique will be employed (Palangio et al., 2007b ${ }^{2}$ ). Combining the simultaneous observations of the electromagnetic field obtained in each network station, we will be able to obtain detailed information about the investigated electromagnetic sources.

\section{Analysis of the signals originated in the Earth's inte- rior}

Concerning to the systematic elaboration of the MEM data set, actually we are focussed on the lower part $[0.001-0.5] \mathrm{Hz}$

\footnotetext{
${ }^{2}$ Palangio, P., Masci, F., Di Persio, M., and Di Lorenzo, C.: Electromagnetic fields measurements in ULF-ELF-VLF $[0.001 \mathrm{~Hz}-$ $100 \mathrm{KHz}$ ] bands, submitted, Adv. Geosc., $2007 \mathrm{~b}$.
}

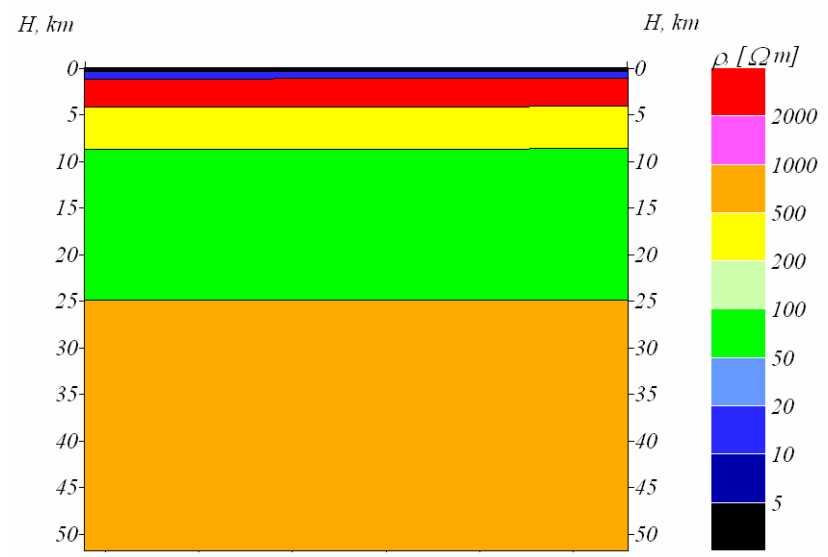

Fig. 5. The Earth's crust resistivity profile calculated in the area of the L'Aquila Observatory. $H$ represents the depth under the site.

of the investigated band. Rare elaborations have been carried out using the high frequency signals. The electric conductivity of the rocks is an important physical property of the Earth's crust. Its time variation can be related to the geodynamical processes and can be monitored by means of the study of the magnetic transfer functions variation, and therefore by the associated magnetic induction vectors variation. So, the transfer functions monitoring is an available method for the crustal conductivity changes investigation in the area of the measurement site and therefore it could be an important research tool in the earthquakes prediction. As example, in Fig. 5 is reported the ground electric resistivity profile calculated for L'Aquila station by means of the single station magnetotelluric tensor evaluation. The resistivity profile has been obtained using the conventional MT approach. The knowledge of the ground resistivity profile is an essential requirement to study the electromagnetic manifestations linked to the earthquakes. The single-station transfer function method is based on the time variation of the three geomagnetic components measured in the same place. If $\mathrm{Z}$, $H$ and $D$ are respectively the variations of the vertical component and the north and east horizontal components of the geomagnetic field, we can assume, in the frequency domain, the relation

$\mathrm{Z}(\omega)=\mathrm{A}(\omega) \mathrm{H}(\omega)+\mathrm{B}(\omega) \mathrm{D}(\omega)$

where $\mathrm{A}(\omega)$ and $\mathrm{B}(\omega)$ are the magnetic transfer functions and $\omega$ is the frequency. $\mathrm{A}(\omega)$ and $\mathrm{B}(\omega)$ are generally complex functions. They are time invariant if the electric resistivity of the ground doesn't change, that is when no large crustal changes due to geodynamical processes are in progress. Starting from $\mathrm{A}(\omega)$ and $\mathrm{B}(\omega)$, we can define a pair of induction vectors, each corresponding to the real and the imaginary components of the transfer functions. The magnitudes of the 

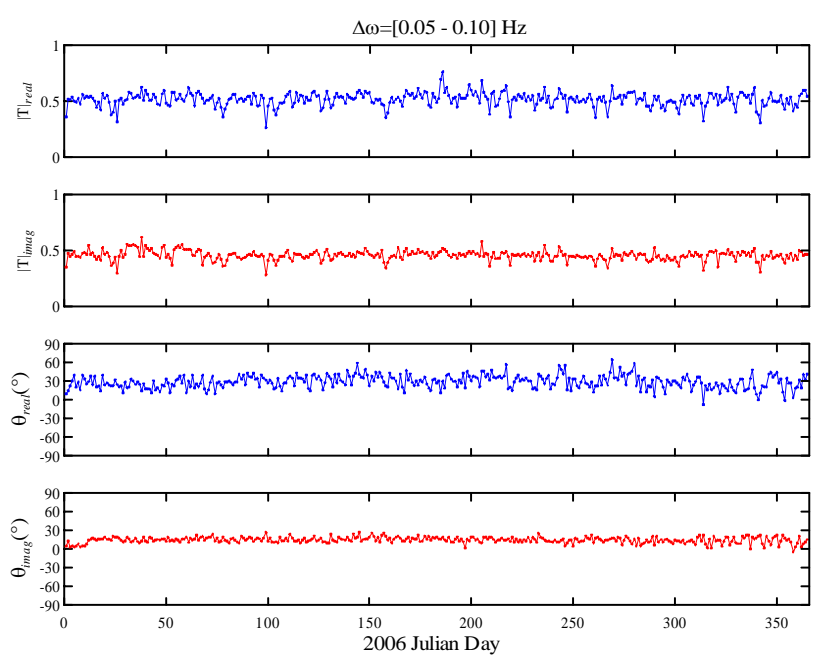

Fig. 6. Daily means of the real and imaginary magnetic induction vectors calculated for the data collected during 2006 in the L'Aquila MEM site in the frequency band $[0.05-0.1] \mathrm{Hz}$. This frequency interval is related to the crustal depth within $25 \mathrm{~km}$.

real and imaginary induction vectors are:

$|\mathrm{T}(\omega)|_{\text {real }}=\sqrt{\mathrm{A}_{\text {real }}(\omega)^{2}+\mathrm{B}_{\text {real }}(\omega)^{2}}$

$|\mathrm{T}(\omega)|_{\text {imag }}=\sqrt{\mathrm{A}_{\text {imag }}(\omega)^{2}+\mathrm{B}_{\text {imag }}(\omega)^{2}}$

whereas the corresponding real and imaginary phases are

$\Theta(\omega)_{\text {real }}=\tan ^{-1}\left[\mathrm{~B}_{\text {real }}(\omega) / \mathrm{A}_{\text {real }}(\omega)\right]$

$\Theta(\omega)_{\text {imag }}=\tan ^{-1}\left[\mathrm{~B}_{\text {imag }}(\omega) / \mathrm{A}_{\text {imag }}(\omega)\right]$

In Fig. 6 is reported the time variation of the magnetic induction vectors calculated for the whole 2006 data set of L'Aquila MEM site in the frequency band $[0.05-0.10] \mathrm{Hz}$. The considered frequency interval can be related to the crustal depth within the first $25 \mathrm{~km}$. In Central Italy the major tectonic activity is concentrated at an underground depth range of about [5-15] $\mathrm{km}$. In this area the direction of the active faults is roughly NW-SE. In Fig. 6 can be noted that the direction of the real vector points toward the tectonic alignment, perpendicularly to the breaking planes of the faults structure. The imaginary vector has the same direction of the real vector. This suggests almost a $2 \mathrm{D}$ resistivity structure in the middle part of the crust. In Fig. 7 is reported the time variation of the magnetic induction vectors in the frequency band $[0.001-0.010] \mathrm{Hz}$. This frequency interval can be related to the deeper part of the crust. The figure shows that in this band the directions of the real and imaginary vectors are quite different respect to their directions in the band previously reported. This feature reflects a different orientation of the lateral discontinuity of the resistivity structure. In any case, the real and the imaginary vectors
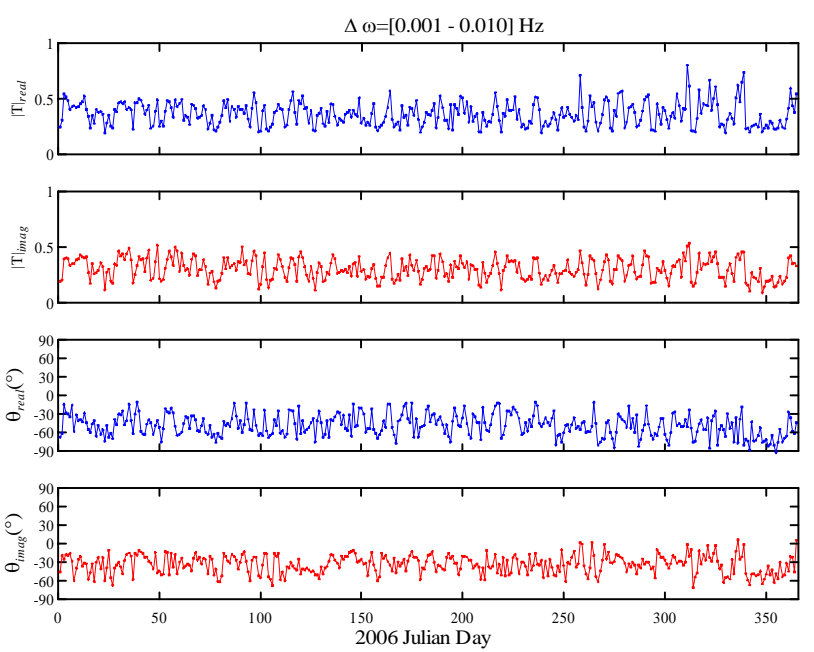

Fig. 7. Daily means of the real and imaginary magnetic induction vectors calculated for the data collected during 2006 in the L'Aquila MEM site in the frequency band $[0.001-0.01] \mathrm{Hz}$. This frequency interval is related to the deeper crust.

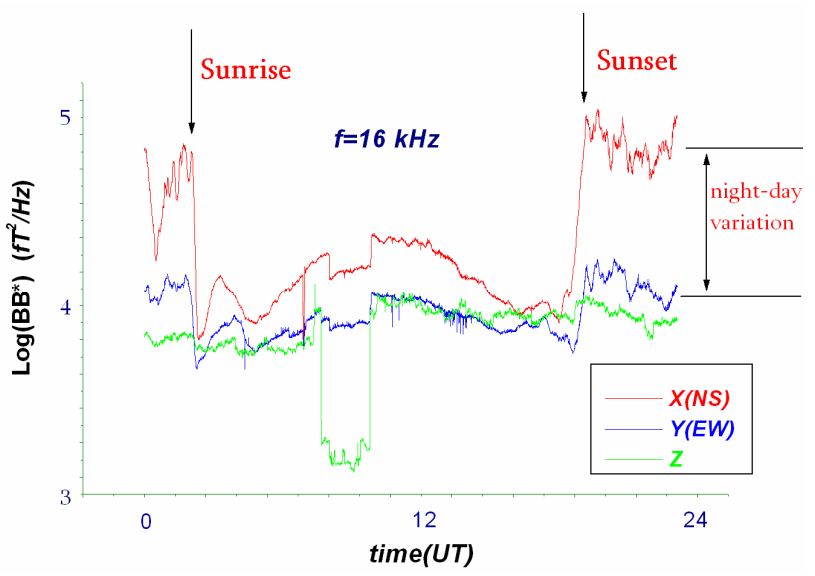

Fig. 8. Large electromagnetic anomaly detected in the L'Aquila MEM station on 14 March 2006 between 08:00 UT and 10:00 UT. The bandwidth is $6 \mathrm{kHz}$ centred around $16 \mathrm{kHz}$. B represents each of the three magnetic field components $(X, Y, Z)$. The anomaly is probably due to a local electromagnetic source.

are parallel each other; this clearly shows again a 2D resistivity structure in the lower part of the crust, but different respect to the resistivity structure of the middle part of the crust. The induction vectors reported in Figs. 6 and 7 do not show anomalous behaviour during the reported time period. Anyway no significant seismic activity has been registered in Central Italy during 2006, so no significant anomaly in the local geomagnetic field is expected. Some studies report magnetic anomalies related to the seismic activity in a band of frequencies extremely wide, extended from $\mathrm{mHz}$ to MHz. In Fig. 8 is reported an example of a large electro- 
magnetic anomaly occurred in the L'Aquila MEM station on 14 March 2006 between 08:00 UT and 10:00 UT. The plot shows the three components of the magnetic field in a band wide $6 \mathrm{kHz}$ around $16 \mathrm{kHz}$. This anomaly cannot be generated or reflected by the ionosphere because the magnetic field components do not fulfil the Earth's boundary conditions. Moreover, the anomaly cannot be generated in the Earth's interior because the skin depth is less than the thickness of the high conductivity sheet in the superficial part of the crust (see Fig. 5). This anomaly is probably due to a local electromagnetic source. In Fig. 9 is reported an example of the residual vertical magnetic field $\Delta Z$ obtained by the following expression

$$
\Delta Z(t)=Z_{\mathrm{mis}}(t)-\left[I_{1}(t) \otimes H(t)+I_{2}(t) \otimes D(t)\right]
$$

In the Eq. (6) the residual vertical magnetic field is the difference between the vertical component $Z_{\text {mis }}$ of the measured magnetic field and the vertical component of the synthetic magnetic field evaluated by the convolution of the horizontal magnetic field components $H$ and $D$ with the Earth's impulse response functions $I_{1}$ and $I_{2}$ (Palangio et al., 2007a ${ }^{1}$ ). The difference between the two signals could be due to the sources located in the Earth interior.

\section{Conclusions}

The scientific and the technological objectives of the MEM project are reported particularly concerning to the monitoring of the electromagnetic signals originated in the Earth's interior. We have also reported some results obtained in the first MEM station installed in the area of the INGV Observatory of L'Aquila. Using the single station magnetotelluric approach we have obtained some valuables information about the underground resistivity structure in the area of the station. Concerning to the study of the magnetic signals linked to the tectonic activity we have reported the behaviour of the magnetic induction vectors characteristics in the lower frequency band $[0.001-0.1] \mathrm{Hz}$ for the whole 2006. This analysis shows the normal behaviour of the magnetic induction vectors when no large crustal changes due to geodynamical processes are present. In the future this kind of analysis will be pursued for a longer period of time in order to understand the relationships between the possible anomalous behaviours of the magnetic induction vectors and the parameters of the earthquakes such as the magnitude, the depth of the hypocenter and its distance from the measurement site.

Acknowledgements. The authors thank the staff of the L'Aquila Observatory for the basic support in the research activity. This work was supported by the MEM Project (Interreg IIIA Adriatic Cross Border Programme).

Edited by: M. Contadakis

Reviewed by: O. Molchanov and another anonymous referee

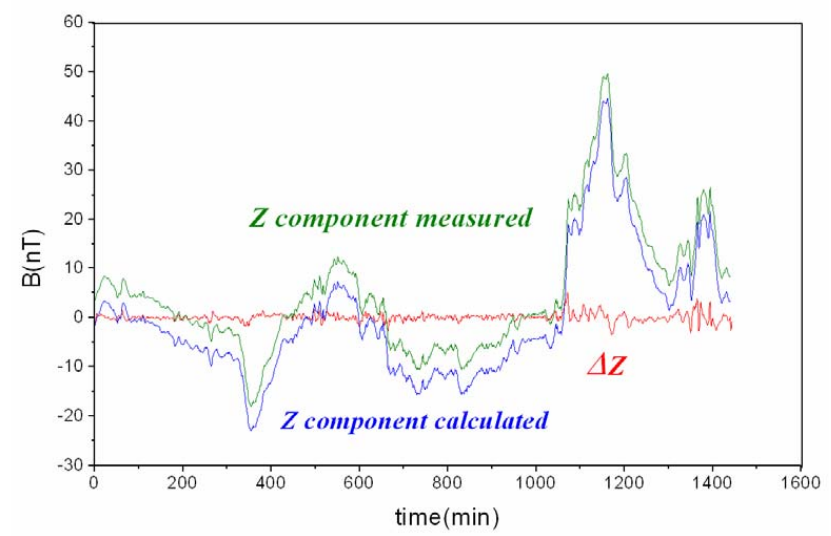

Fig. 9. The residual vertical magnetic field $\Delta Z$ obtained by the Eq. (6). The vertical scale corresponds only to the difference $\Delta Z$. The measured and the synthetic vertical components shown in figure are scaled for a best understanding.

\section{References}

Frid, V., Bahat, D., Goldbaum, J., and Rabinovich, A.: Experimental and theoretical investigations of electromagnetic radiation induced by rock fracture, Israel J. Earth Sci., 49, 9-19,2000.

Kawate, R., Molchanov, O. A., and Hayakawa, M.: Ultralowfrequency magnetic fields during the Guam earthquake of 8 August 1993 and their interpretation, Phys. Earth Planet. In., 105, 229-238, 1998.

Johnston, M. J. S.: Precision of magnetic measurements in a tectonically active region, J. Geomag. Geoelect., 36, 83-95, 1984.

Lanzerotti, L. J., Maclennan, C. G., and Fraser-Smith, A. C.: Background magnetic spectra: $\sim 10 \mathrm{E}-5$ to $\sim 10 \mathrm{E}+5 \mathrm{~Hz}$, Geophys. Res. Lett., 17, 10, 1593-1596, 1990.

Molchanov, O. A., Kopytenko, Y. A., Voronov, P. M., Kopytenko, E. A., Matiashvili, E. A., Fraser-Smith, A. C., and Bernardi, A. : Results of ULF magnetic field measurements near the epicenters of the Spitak $(\mathrm{Ms}=6.9)$ and Loma Prieta $(\mathrm{Ms}=7.1)$ earthquakes comparative analysis, Geophys. Res. Lett., 19, 1495-1498, 1992.

Molchanov, O. A., and Hayakawa, M.: Generation of ULF electromagnetic emissions by microfracturing, Geophys. Res. Lett., 22, 3091-3094, 1995.

Palangio, P.: Radioricezione ELF-VLF, Ann. Geofis., 36, 5-6, 99114, 1993.

Parrot, M., Achache, J., Berthelier, J. J., Blanc, E. , Deschamps, A., Lefeuvre, F., Menvielle, M., Plantet, J. L., Tarits, P., and Villian, J. P.: High-frequency seismo-electromagnetic effects, Phys. Earth Planet. Int., 77, 65-83, 1993.

Spitz, S.: The magnetotelluric impedance tensor properties with respect to rotations, Geophysics, 50, 10, 1610-1617, 1985.

Teisseyre, R., and Ernst, T.: Electromagnetic radiation related to dislocation dynamics in a seismic preparation zone, Ann. GeophItaly, 45, 393-399, 2002. 